\title{
Magnetic island deformation due to sheared flow and viscosity
}

\author{
C. $\operatorname{Ren}^{\mathrm{a})}$ \\ University of Wisconsin-Madison, Madison, Wisconsin 53706-1687 \\ M. S. Chu \\ General Atomics, P.O. Box 85608, San Diego, California 92186-9784 \\ J. D. Callen \\ University of Wisconsin-Madison, Madison, Wisconsin 53706-1687
}

(Received 20 November 1998; accepted 23 December 1998)

\begin{abstract}
A partial differential equation for a two-dimensional magnetohydrodynamic (MHD) equilibrium with flow and viscosity is derived. The equation is used to describe the deformation of the magnetic island caused by the viscous drag of a sheared flow. The deformation is characterized by the phase gradient of the magnetic perturbation across a magnetic island. This phase gradient has been observed experimentally in electron cyclotron emission data from the DIII-D tokamak [J. L. Luxon and L. G. Davis, Fusion Technol. 8, 441 (1985)]. (C) 1999 American Institute of Physics.
\end{abstract}

[S1070-664X(99)02904-3]

\section{INTRODUCTION}

Since plasma flow exists in a wide range of magnetic fusion devices (for example, tokamaks with neutral beam injection), there has been a continual interest in the effects of flow on the MHD (magnetohydrodynamic) equilibrium. A second-order partial differential equation for the poloidal magnetic flux function, similar to the Grad-Shafranov equation for the static case, ${ }^{1}$ can be found for an axisymmetric and nondissipative (zero resistivity and viscosity) equilibrium. ${ }^{2}$ The resistive effect on the equilibrium has been studied in Ref. 3. Hurricane et al. have studied the viscous drag due to the plasma flow on a magnetic island structure in a slab geometry, using an initial-value MHD code. ${ }^{4}$ There, the emphasis was on the exchange of forces between the magnetic field and the plasma.

In this paper, we study the viscous effect on an MHD equilibrium with flow. In particular, we show the deformation of a magnetic island structure by the combined effects of the sheared flow and viscosity. The main motivation of the present work comes from the recent study of the MHD mode structure in tokamaks using the ECE (electron cyclotron emission) diagnostic, ${ }^{5}$ which reveals deviations of the magnetic island structure from the usual symmetric (left-right symmetric about the rational surface and up-down about the O-point) case. Figure 1 is a sketch of the magnetic flux contours for a normal magnetic island (a) and a deformed one (b). A central result of this paper is the derivation of a GradShafranov-like equation for the MHD equilibrium with flow and viscosity, in a two-dimensional slab geometry with the third direction being the symmetry direction. The viscous term is shown to have the needed symmetry property to induce the island deformation observed in the experiment. The island deformation can be quantitatively related to the

\footnotetext{
${ }^{a)}$ Current address: Department of Physics and Astronomy, UCLA, Los Angeles, California 90095
}

sheared flow and viscosity by treating the viscous term as a perturbation when solving the equation for the equilibrium.

The paper is organized as follows. The derivation of the equilibrium equation is given in Sec. II. An analytically solvable model is presented in Sec. III to give a scaling formula for the magnitude of the island deformation. Numerical solutions of the equilibrium equation are presented in Sec. IV. Experimental evidence for a deformed magnetic island structure from the DIII-D tokamak ${ }^{6}$ is presented in Sec. V. Finally, a short discussion is given in Sec. VI.

\section{BASIC EQUATIONS}

We start with the equilibrium momentum balance equation as our basic equation:

$$
\rho \mathbf{V} \cdot \nabla \mathbf{V}=-\nabla p+\mathbf{j} \times \mathbf{B}+\nu \nabla^{2} \mathbf{V}+\mathbf{S}_{V},
$$

together with the ideal Ohm's law:

$$
-\nabla \Phi+\mathbf{V} \times \mathbf{B}=0 .
$$

Here, the vector functions $\mathbf{V}, \mathbf{j}$, and $\mathbf{B}$ are the velocity, current density, and magnetic field respectively, and the scalar functions $\rho, p$, and $\Phi$ are the mass density, pressure, and electrostatic potential, respectively. For simplicity, we assume spatially constant mass density. A constant viscosity is represented by $\nu$. Finally, $\mathbf{S}_{V}$ represents an external momentum source, which is necessary to achieve steady state when viscous dissipation is present.

We use a Cartesian coordinate in which the $\mathbf{z}$-direction is taken to be the symmetry direction $(\partial / \partial z=0)$. Then, the magnetic field can be expressed in terms of its $z$-component $B_{z}$ and a magnetic flux function $\Psi$ :

$$
\mathbf{B}=B_{z} \mathbf{z}+\mathbf{z} \times \nabla \Psi .
$$

Because of the symmetry, the $z$-component of the flow velocity will not affect the magnetic flux function $\Psi$. There- 

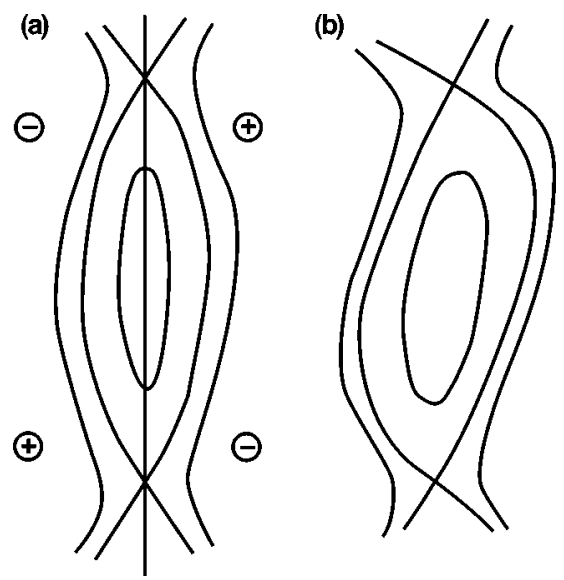

FIG. 1. (a) Sketch of the magnetic flux contours $\left(\Psi_{0}\right)$ of an unperturbed magnetic island. Shown also is the sign of the viscous force in the $\nabla \Psi_{0}$-direction induced by the plasma flow. (b) Sketch of the magnetic flux contours of the deformed magnetic island.

fore, we only consider the flow velocity in the $x y$-plane. With the incompressibility assumption, the flow velocity can be written as

$$
\mathbf{V}=\mathbf{z} \times \nabla U .
$$

Here, $U$ is the stream function.

With $\mathbf{B}$ and $\mathbf{V}$ expressed in this form, the ideal Ohm's law becomes

$$
-\nabla \Phi+B_{z} \nabla U+\mathbf{z}(\mathbf{z} \cdot \nabla U \times \nabla \Psi)=0 .
$$

The symmetry in the $\mathbf{z}$-direction requires that $U$ be a function of $\Psi$. It then follows that $\Phi$ and $B_{z}$ are all functions of $\Psi$. Thus, we have $\Phi=\Phi(\Psi), B_{z}=B_{z}(\Psi)$, and $U=U(\Psi)$.

We next look at each of the three components- $\mathbf{z}, \mathbf{z}$ $\times \nabla \Psi$, and $\nabla \Psi$ - of the momentum Eq. (1). The $z$-component is decoupled from the other two components and is simply $\mathbf{S}_{V_{z}}=0$.

The $\mathbf{z} \times \nabla \Psi$-component of the equation is

$$
-\mathbf{B}_{p} \cdot \nabla\left(\frac{p+\rho V^{2}}{2}\right)+\nu \nabla \Psi \cdot \nabla\left(\nabla^{2} U\right)+\mathbf{B}_{p} \cdot \mathbf{S}_{V}=0,
$$

with $\mathbf{B}_{p}=\mathbf{z} \times \nabla \Psi$. Integrating this equation, we obtain a Bernoulli relation:

$$
p+\frac{\rho V^{2}}{2}=H(\Psi)+\int_{0}^{l_{p}} \frac{d l_{p}}{B_{p}}\left[\nu \nabla \Psi \cdot \nabla\left(\nabla^{2} U\right)+\mathbf{B}_{p} \cdot \mathbf{S}_{V}\right],
$$

with $l_{p}$ being the length along a magnetic field line and $H$ an arbitrary function of $\Psi$. This requires the solubility condition $\oint d l_{p} / B_{p}\left[\nu \nabla \Psi \cdot \nabla\left(\nabla^{2} U\right)+\mathbf{B}_{p} \cdot \mathbf{S}_{V}\right]=0$.

To simplify the $\nabla \Psi$-component of the momentum equation, we assume that the momentum source has no $\nabla \Psi$ component: $\nabla \Psi \cdot \mathbf{S}_{V}=0$. We further assume that the part of the enthalpy $\left(p+\rho V^{2} / 2\right)$ that varies within magnetic flux surfaces has no variation in the $\nabla \Psi$-direction, $\nabla \Psi$ $\cdot \nabla \int_{0}^{l_{p}}\left(d l_{p} / B_{p}\right)\left[\nu \nabla \Psi \cdot \nabla\left(\nabla^{2} U\right)+\mathbf{B}_{p} \cdot \mathbf{S}_{V}\right]=0$. With these two assumptions, the $\nabla \Psi$-component of the momentum equation becomes

$$
\nabla^{2} \Psi+F(\Psi)-\mu_{0} \rho U^{\prime} \nabla^{2} U=\mu_{0} \nu \frac{\nabla \Psi \cdot \mathbf{z} \times \nabla\left(\nabla^{2} U\right)}{|\nabla \Psi|^{2}} .
$$

Here, $F(\Psi)=B_{z} B_{z}^{\prime}+\mu_{0} H^{\prime}$ and $\mu_{0}$ is the vacuum permeability.

Equation (6) is a generalized equation for an MHD equilibrium with flow and viscosity, with three free magnetic flux surface functions $B_{z}, H$, and $U$. Usually the plasma viscosity is small and the viscous term on the right of Eq. (6) can be treated as a perturbation. Using $\nabla^{2} U=U^{\prime \prime}(\nabla \Psi)^{2}+U^{\prime} \nabla^{2} \Psi$ and $\Psi$, Eq. (6) can be written in a relatively more transparent form:

$$
\begin{gathered}
\left(1-\mu_{0} \rho U^{\prime 2}\right) \nabla^{2} \Psi+F(\Psi)-\mu_{0} \rho U^{\prime} U^{\prime \prime}(\nabla \Psi)^{2} \\
=\mu_{0} \nu U^{\prime \prime} \frac{\nabla \Psi \cdot \mathbf{z} \times \nabla|\nabla \Psi|^{2}}{|\nabla \Psi|^{2}}+O\left(\nu^{2}\right) .
\end{gathered}
$$

We assume that $\left(1-\mu_{0} \rho U^{\prime 2}\right)$ is positive in the entire region and there is no Alfvén resonance present.

In the remainder of the paper we will use Eq. (7) to study the deformation of a magnetic island due to the viscous force. Using an unperturbed magnetic island for the $\Psi$-function in the viscous term, the general symmetry properties of the deformation can be obtained. The perturbation has an odd symmetry, both relative to the vertical axis at the rational surface and the horizontal axis across the O-point [Fig. 1(a)]. The general shape of the resultant deformed island is also sketched in Fig. 1(b). Analytical and numerical evaluations of the deformation will be presented in Sec. III and Sec. IV, respectively.

\section{AN ANALYTICALLY SOLVABLE EXAMPLE}

We use a simple analytically solvable model to illustrate the dependence of the magnetic island structure on the flow shear and viscosity. We assume that the flow shear $\left(U^{\prime \prime}\right)$ and viscosity are small. Therefore, the viscous term and the $(\nabla \Psi)^{2}$-term in Eq. (7) can be treated as perturbations. We write $\Psi=\Psi_{0}+\Psi_{1}$, where the perturbed part $\Psi_{1}$ is much smaller than the unperturbed part $\Psi_{0}$. Then, the equation for $\Psi_{0}$ is

$$
\nabla^{2} \Psi_{0}+f\left(\Psi_{0}\right)=0
$$

where $f\left(\Psi_{0}\right)=F\left(\Psi_{0}\right) /\left(1-\mu_{0} \rho U^{\prime 2}\right)$.

Consider a simple case of constant $f, f=-\mu_{0} j_{0}$. A solution for $\Psi_{0}$, which possesses a magnetic island with a wave number $k$ and half-width $w$, can be found:

$$
\Psi_{0}=\Psi_{c}\left[\left(\frac{x}{w}\right)^{2}-\frac{1+\cos k y}{2}\right] .
$$

Here, $\Psi_{c}=\mu_{0} j_{0} w^{2} / 2$ and a thin island approximation, $k w$ $\ll 1$, has been used. The value of $\Psi_{0}$ for the separatrix of the island is $\Psi_{\text {sep }}=0$.

With the $\Psi_{0}$ given in Eq. (8), the source term for calculating the perturbation $\Psi_{1}$ has many harmonics. We are interested in perturbations whose $y$-dependence is sin $k y$ and is odd in $x$. This kind of perturbation will produce a phase gradient in the unperturbed part [Eq. (8)], which has been found in the experimental data. ${ }^{5}$ The source for this particu- 
lar form of perturbation comes from the viscous term in Eq. (7). Therefore, using Eq. (8) for $\Psi_{0}$, the equation for $\Psi_{1}$ can be written as

$$
\nabla^{2} \Psi_{1}=\mu_{0} \nu\left(\frac{k w U^{\prime \prime} \Psi_{c} / w^{2}}{1-\mu_{0} \rho U^{\prime 2}}\right) \frac{(x / w) \sin k y}{(x / w)^{2}+[(k w / 4) \sin k y]^{2}} .
$$

Suppose the flow shear is concentrated around the separatrix and can be modeled by a $\delta$-function, $U^{\prime \prime}=U_{\text {sep }}^{\prime} \delta(\Psi)$. Then, writing $\Psi_{1}=\Psi_{1}(x) \sin k y$, multiplying both sides of Eq. (9) by $\sin k y$, and integrating over $y$, we obtain an equation for $\Psi_{1}(x)$ :

$$
\Psi_{1}^{\prime \prime}(\zeta)=A \sqrt{1-\zeta^{2}}
$$

when $\zeta<1$; and

$$
\Psi_{1}^{\prime \prime}(\zeta)=0
$$

when $\zeta>1$. Here, $\zeta \equiv x / w, \quad A \equiv(4 / \pi) \mu_{0} \nu k w U_{\text {sep }}^{\prime}\langle 1 /(1$ $\left.\left.-\rho \mu_{0} U^{\prime 2}\right)\right\rangle$ and the thin island approximation has been used. Since a jump of $U^{\prime}$ is assumed across the separatrix, $\langle\cdots\rangle$ indicates the expression should be evaluated using some averaged value of $U^{\prime}$ near the separatrix.

Motivated by the experimental measurement to be described in Sec. V, we use the following boundary conditions for $\Psi_{1}: \Psi_{1}(0)=0$ and $\Psi_{1}^{\prime}(\zeta>1)=$ const. Then, the solution is

$$
\Psi_{1}=\frac{A}{2}\left(\zeta \sin ^{-1} \zeta+\frac{2+\zeta^{2}}{3} \sqrt{1-\zeta^{2}}-\frac{3}{2}\right)
$$

for $\zeta<1$ and

$$
\frac{\pi}{4} A\left(\zeta-\frac{4}{3 \pi}\right)
$$

for $\zeta>1$. The effect of this perturbation is to add a phase to $\Psi_{0}$ and the total magnetic flux function can be written as $\Psi=\Psi_{c} \zeta^{2}-1 / 2-1 / 2 \cos \{[k y+\theta(\zeta)]\}, \quad$ where $\theta(\zeta)$ $=2 \Psi_{1}(\zeta) / \Psi_{c}$. The form of the perturbation $\Psi_{1}$ predicts that the phase has a constant gradient outside the island $(\zeta$ $>1)$ :

$$
\frac{d \theta}{d \zeta}=\frac{\pi A}{\mu_{0} j_{0} w^{2}}
$$

This phase gradient can also be written in terms of the relevant physical quantities as

$$
\frac{d \theta}{d x}=4\left[\frac{\rho(k \nu / \rho) V^{\prime}}{B^{2} / \mu_{0}}\right] \text {. }
$$

Here, $V^{\prime}$ and $B$ are the flow velocity shear and "perpendicular' magnetic field at the separatrix.

\section{NUMERICAL RESULTS}

In this section, we numerically solve Eq. (7), by prescribing more complete forms for the free functions $F$ and $U$, and retaining all the harmonics from the viscous term. It will be shown that the essential features of the analytical solutions are preserved under the more accurate treatment.

We start with an equilibrium with flow but negligible viscosity $(\nu=0)$ and treat the viscous term as a perturbation.
We decompose the total flux function into two parts: $\Psi$ $=\Psi_{0}+\Psi_{1}$, with $\Psi_{1} \ll \Psi_{0}$. The equation for $\Psi_{0}$ is

$$
\nabla^{2} \Psi_{0}+\frac{F\left(\Psi_{0}\right)-\epsilon U^{\prime} U^{\prime \prime}\left|\nabla \Psi_{0}\right|^{2}}{1-\epsilon U^{\prime 2}}=0 .
$$

Here, the dimensionless number $\epsilon \equiv \rho V^{2} /\left(B^{2} / \mu_{0}\right)$ represents the flow magnitude relative to the magnetic field ( $V$ and $B$ are the typical flow velocity and magnetic field) and corresponds to a flow Mach number.

The equation for $\Psi_{1}$, the perturbed flux, can be obtained by linearizing Eq. (7):

$$
\nabla^{2} \Psi_{1}+R\left(\Psi_{0}\right) \Psi_{1}+T\left(\Psi_{0}\right) \nabla \Psi_{0} \cdot \nabla \Psi_{1}=\nu_{n} S\left(\Psi_{0}\right),
$$

with $R, S$, and $T$ calculated from $\Psi_{0}$,

$$
\begin{aligned}
R\left(\Psi_{0}\right)= & \frac{2 \epsilon U^{\prime} U^{\prime \prime}\left[F\left(\Psi_{0}\right)-\epsilon U^{\prime} U^{\prime \prime}\left|\nabla \Psi_{0}\right|^{2}\right]}{\left(1-\epsilon U^{\prime 2}\right)^{2}} \\
& +\frac{\left[F^{\prime}-\epsilon\left|\nabla \Psi_{0}\right|^{2}\left(U^{\prime} U^{\prime \prime \prime}+U^{\prime \prime 2}\right)\right]}{\left(1-\epsilon U^{\prime 2}\right)}, \\
T\left(\Psi_{0}\right)= & -\frac{2 \epsilon U^{\prime} U^{\prime \prime}}{\left(1-\epsilon U^{\prime 2}\right)},
\end{aligned}
$$

and

$$
S\left(\Psi_{0}\right)=\frac{2 U^{\prime \prime}}{\left(1-\epsilon U^{\prime 2}\right)^{2}} \frac{\nabla \Psi_{0} \cdot \mathbf{z} \times \nabla\left(\left|\nabla \Psi_{0}\right|\right)}{\left|\nabla \Psi_{0}\right|} .
$$

Here, $\nu_{n}$ is the normalized viscosity, $\nu_{n} \equiv(\nu V / w) /\left(B^{2} / \mu_{0}\right)$. The inhomogeneous part $S$ acts as the source for the perturbation. As stated before, it has the proper symmetry. It is also proportional to $U^{\prime \prime}$, the flow shear.

We use the following forms for $F$ and $U^{\prime}$ :

$$
F=a+b \Psi
$$

and

$$
U^{\prime}=\tanh \left[\beta\left(\Psi_{s}-\Psi\right)\right],
$$

with $a, b, \beta$, and $\Psi_{s}$ being four constant parameters. We usually choose $\Psi_{s}$ to be near the separatrix of the island so that the flow shear is concentrated near the separatrix.

The equations are solved in a rectangular region. The dimension in the $y$-direction is one wavelength of the island and the dimension in the $x$-direction is about 10 island halfwidths. The rational surface is located at the left boundary. Therefore, we only solve for one side of the island. The other side can be easily reconstructured by symmetry.

To introduce the initial island configuration, we use the following boundary condition for $\Psi_{0}: \Psi_{0 R}=0$ at the right boundary and $\Psi_{0 L}=1 / 2-w^{2} / 4 \cos (k y)$, where $k$ and $w$ are the wave number and half-width of the island. The boundary condition for $\Psi_{1}$ at the left boundary is $\Psi_{1 L}=0$ (no perturbation at the rational surface). The boundary condition for $\Psi_{1}$ at the right boundary is prescribed according to the expected physical condition. If there is another rational surface nearby and the modes are coupled, then $\Psi_{1 R}$ should be equal to a prescribed value. If there is no mode-coupling and the right boundary is sufficiently far away from the rational sur- 

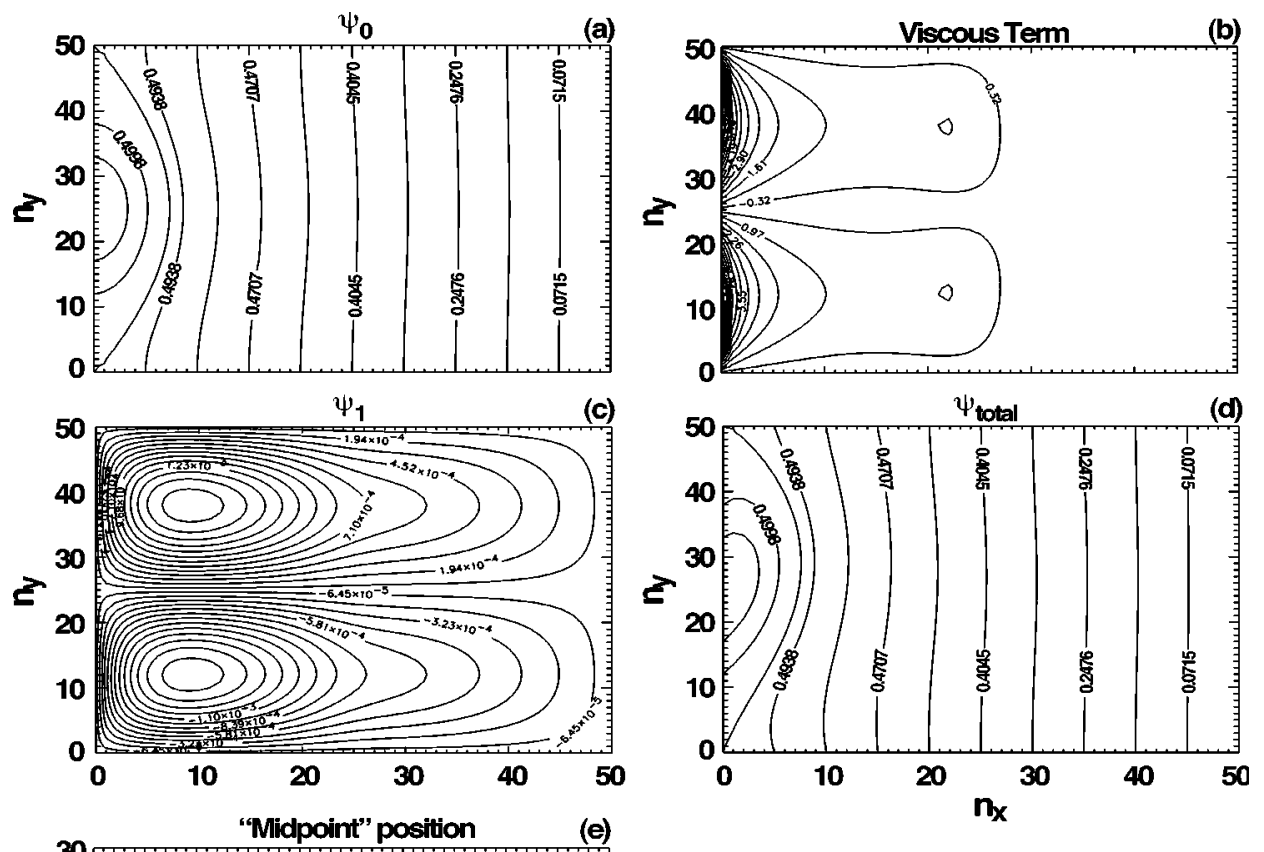

FIG. 2. (a) Contour plot of $\Psi_{0}$ : the unperturbed island. (b) Contour plot of the viscous term $\left(S / v_{n}\right)$. Notice the odd symmetry about the midplane located at $n_{y}=25$. (c) Contour plot of $\Psi_{1}$, the perturbed magnetic flux. It is also antisymmetric with respect to the midplane. (d) Contour plot of $\Psi_{0}$ $+\Psi_{1}$. This shows an island deformed by the shear flow in the presence of viscosity. (e) The position of the points where $d \Psi / d y=0$ in the deformed island. For an unperturbed island, those points should be at the midplane $\left(n_{y}\right.$ $=25$ ). face, the proper boundary condition should be $\Psi_{1 R}=0$. Here we use the latter. We use periodic boundary conditions in the $y$-direction for both $\Psi_{0}$ and $\Psi_{1}$.

We solve Eqs. (11) and (12) on a $51 \times 51$ grid. The lengths in the $x$-and $y$-directions are 1.0 (with $w=0.12$ ) and 4.189 , respectively. The numerical values used to represent $F$ and $U^{\prime}$ are: $a=-0.42356, b=2.01, \quad \beta=20$, and $\Psi_{s}$ $=0.495$. With this value of $\beta$, the flow shear is quite high and well localized around the separatrix. The two dimensionless parameters are taken to be $\epsilon=0.8$ and $\nu_{n}=0.05$. Shown in Fig. 2(a) is a contour plot of $\Psi_{0}$ [solution to Eq. (11)] in the $x y$-plane. This is an island without the effect of the viscous drag.

In Fig. 2(b) we plot the viscous term [Eq. (13)]. This term shows the expected odd symmetry about the mid-plane at $n_{y}=25$. It also becomes small away from the separatrix, where the field lines are hardly bent and there is almost no flow shear.

The contour plots of the perturbation $\Psi_{1}$ and the total flux magnetic flux function $\Psi=\Psi_{0}+\Psi_{1}$ are plotted in Figs. 2(c) and 2(d). We can see the deformed island structure in Fig. 2(d). To obtain the phase gradient outside the island, which can be measured in the experiment, we plot the $y$ coordinate of the points where $d \Psi / d y=0$ in Fig. 2(e). In an unperturbed island [Fig. 2(a)], these points are all in the midplane $\left(n_{y}=25\right)$. The phase gradient outside the island in this case is 12 degree/half-island-width [Fig. 2(e)].

\section{EXPERIMENTAL EVIDENCE}

The magnetic island structure has also been studied experimentally using the ECE (electron cyclotron emission) diagnostic. ${ }^{5,7,8}$ The ECE diagnostic measures the local electron temperature $T_{e}$, which is assumed to be constant on magnetic surfaces due to the very high electron heat conductivity along the magnetic field lines. As the plasma rotates by the fixed ECE diagnostic, $T_{e}$ at different toroidal locations is recorded by ECE channels. When there is a magnetic island, the magnetic surfaces contract and expand periodically along the toroidal direction. Thus, an ECE channel corresponding to a given major radius can measure $T_{e}$ on different magnetic surfaces. Therefore, the measured $T_{e}$ from an ECE channel shows periodic temporal fluctuations. The phase gradient calculated in Eq. (10) and in the numerical solution plotted in Fig. 2(e) is directly related to the relative phases of the fluctuations between the channels.

Plotted in Fig. 3 is the measured phases of the electron temperature fluctuations from the DIII-D tokamak. ${ }^{6}$ The magnetic island can be identified from the large (nearly 160 degree) phase jump near $R=2.05 \mathrm{~m}$, where the rational surface is located. The deformation of the island can be seen from the gradients of the phases on the two sides of the rational surface. (An unperturbed and symmetric island would render two constant phases, with 180 degree difference, on both sides of the rational surface, like a step.) Fur- 


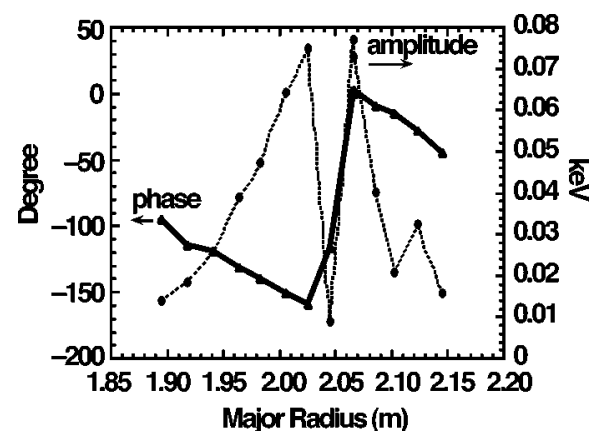

FIG. 3. Phase and amplitude profile of the electron temperature fluctuations measured by ECE for DIII-D Shot 92515 . The width for this $3 / 2$ island can be determined from the two peaks of the amplitude. The rational surface is near the minimum amplitude. Notice the phase gradient on both sides of the rational surface.

thermore, the phase gradient has the same sign on both sides, which is consistent with the deformation illustrated in Fig. 1(b).

The phase gradient in Fig. 3 is estimated to be 9 degree/ half-island-width, which is on the same order of magnitude as the value of 12 degree/half-island-width in our numerical example. This indicates that the values for $\epsilon$ and $\nu_{n}$ used for Fig. 2 are reasonable for representing experimental situation. Since we do not have a direct measurement of the viscosity and the relevant component of magnetic field and flow velocity, it is difficult to obtain experimental values for $\epsilon$ and $\nu_{n}$. However, an order-of-magnitude estimate can be made to show that the values used in the numerical simulation are reasonable. For example, let us assume the density of a deuterium plasma is $5 \times 10^{19} \mathrm{~m}^{-3}$, the anomalous specific viscosity $\nu / \rho=1 \mathrm{~m}^{2} / \mathrm{s}$, the wave number of the island $k$ $=7 \mathrm{~m}^{-1}$, and the typical magnitude of flow velocity and magnetic field in the plane perpendicular to the symmetry direction are $V=10^{3} \mathrm{~m} / \mathrm{s}$ and $B=5$ Gauss, respectively. Then, the two dimensionless parameters have the same values as in the numerical simulation in Sec. IV, i.e., $\epsilon=0.8$ and $\nu_{n}=0.05$. Notice that the relevant flow velocity here is to be measured in a frame that is rotating with the magnetic island.

\section{DISCUSSION}

The slab geometry used in this paper is a simplification of the tokamak configuration. In a tokamak, the plasma flow is mainly in the toroidal direction. The helical symmetry direction of the magnetic island is a combination of the toroi- dal and poloidal directions. It is the flow component perpendicular to the helical symmetry direction that causes the island structure deformation. Furthermore, in the helical symmetry case, the component of the momentum equation that is parallel to the symmetry direction is no longer separated from the other components. Extending the slab model to a more realistic helical geometry will be the subject of future work. However, we expect that the basic features of the deformation will be preserved.

The zeroth-order solution used in this paper has a magnetic island. However, the method developed here is equally well suited to calculate the deformation of an ideal MHD mode which has no island. Then, there is no 180-degree phase shift across the rational surface; nontheless there can be a phase gradient within the plasma.

The flow pattern is assumed to be antisymmetric relative to the rational surface. The measured phase gradient pattern confirms this assumption (Fig. 3). Symmetric flow patterns, requiring the flow velocity to be zero within the separatrix, would produce a different ECE fluctuation phase pattern.

In summary, a partial differential equation for a twodimensional MHD equilibrium with flow and viscosity is derived. The equation is used to describe the deformation of the magnetic island caused by the viscous drag of the sheared flow. The deformation can be characterized by a phase gradient in the magnetic island flux function. This phase gradient has been observed in the ECE electron temperature fluctuation data from the DIII-D tokamak.

\section{ACKNOWLEDGMENTS}

One of the authors (C.R.) happily acknowledges the hospitality of General Atomics, where this research was performed. This work is supported by U.S. Department of Energy under Grant Nos. DE-FG02-92ER54139 and DE-FG0392ER54150 and Contract No. DE-AC03-89ER51114.

${ }^{1} \mathrm{H}$. Grad and H. Rubin, in Proceedings of the Second United Nations International Conference on the Peaceful Uses of Atomic Energy, (United Nations, Geneva 1958) Vol. 31, p. 190; V. D. Shafranov, Sov. Phys. JETP 26, 682 (1960).

${ }^{2}$ E. Hameiri, Phys. Fluids 26, 230 (1983).

${ }^{3}$ G. N. Throumoulopoulos, J. Plasma Phys. 59, 303 (1998).

${ }^{4}$ O. A. Hurricane, T. H. Jensen, and A. B. Hassam, Phys. Plasmas 2, 1976 (1995).

${ }^{5}$ C. Ren, Ph.D. dissertation, University of Wisconsin-Madison, 1998.

${ }^{6}$ J. L. Luxon and L. G. Davis, Fusion Technol. 8, 441 (1985).

${ }^{7}$ C. Ren, J. D. Callen, T. A. Gianakon, et al., Phys. Plasmas 5, 450 (1998).

${ }^{8}$ Z. Chang, E. D. Fredrickson, S. H. Batha, et al., Phys. Plasmas 5, 1076 (1998). 